\title{
Bendamustine in Refractory / Relapsed Non Hodgkin Lymphoma
}

\author{
Amine MAF ${ }^{1}$, Saeed MS ${ }^{1}$ (D), Zaky AH ${ }^{1}$ \\ ${ }^{1}$ Department of Medical Oncology, South Egypt Cancer Institute, Assiut University.
}

\begin{abstract}
:
Background and aim: Non-Hodgkin lymphoma (NHL) is the most prevalent hematological malignancy worldwide. Outcome of lymphoma has definitely improved over the last few decades. Nevertheless, notable group of patients may display a poor response to treatment with a true refractoriness or a transient response followed by early relapse which represent approximately one quarter of NHL cases undergoing induction therapy who remains a major cause of morbidity and mortality with only a small percentage will experience prolonged disease-free survival with salvage chemo immunotherapy. Bendamustine had been suggested in previous studies as a second line therapy for relapsed /refractory (RIR) NHL . This study aimed to evaluate the efficacy and safety of using the Bendamustine as single agent in RIR NHL patients.
\end{abstract}

Patients and Methods: A prospective, single arm study that was carried out on twenty five patient diagnosed as RIR NHL who previously received at least one salvage line of chemotherapy. Patient received bendamustine at dose of $120 \mathrm{mg} /$ $\mathrm{m} 2$ in day 1 and 2 every 3 weeks for 3 cycles intravenous with $250 \mathrm{ml}$ saline over 60 minute then reassessed.

Results: Study revealed that overall response rate was $60 \%$ with patients had complete remission (CR) was (20\%), (40\%) achieved partial remission (PR), $(4 \%)$ had stationary disease (SD) and $(36 \%)$ had progressive disease. Overall survival was $72 \%$ at 12 months and $66 \%$ at 24 months. Moreover, median for progression free survival (PFS) is 12 months with range of (2-24) month, PFS was $68 \%$ at 6 months, $63.8 \%$ at 12 months and $63.8 \%$ at 24 months. In our study three $(12 \%)$ patients had no toxicity and $22(88 \%)$ patients developed toxicity. The main toxic effects are haematological. Eighteen $(72 \%)$ patients developed haematological toxicity and four (16\%) developed nonhaematological toxicity. According to haematological toxicity one of them (4\%) had thrombocytopenia grade 1-2. Four (16\%) of them had thrombocytopenia grade 3-4.Two (8\%) patients had neutropenia grade 1-2 (one of them had febrile neutropenia). Seven $(28 \%)$ patients had neutropenia grade 3-4 (three of them had febrile neutropenia. Two $(8 \%)$ patients had anemia grade1-2. Two $(8 \%)$ of them had anemia grade 3-4.

According to patients who had non-haematological toxicity. One (4\%) of them had fatigue grade 3-4. One (4\%) of them had vomiting grade1-2 .Two $(8 \%)$ patients had diarrhea grade 1-2 this showed that bendamustine is tolerated with low haematological and non-haematological toxicity profile.

Conclusion: Bendamustine is suggested to be an effective regimen in treatment of RIR NHL with high response rate and low toxicity profile.

Keywords: Relapsed / Refractory Non-Hodgkin lymphoma, Bendamustine, OS, PFS.
Received: 15 September 2021
Accepted: 11 October 2021

\author{
Authors Information: \\ Maged Abd El-fattah Amine \\ Medical Oncology department, South \\ Egypt Cancer Institute, Assiut \\ University, Assiut, Egypt. \\ email: maged1907@aun.edu.eg
}

Marwa Saber Saeed

Medical Oncology department, South Egypt Cancer Institute, Assiut University, Assiut, Egypt.

Tel.: 01014312584

email: marwasaber@aun.edu.eg marwasaber0607@gmail.com

Amen Hamdy Zaky

Medical Oncology department, South Egypt Cancer Institute, Assiut University, Assiut, Egypt.

email: amenzaky74@aun.edu.eg

\section{Corresponding Author:}

Marwa Saber Saeed

Medical Oncology department, South

Egypt Cancer Institute, Assiut University, Assiut, Egypt.

Tel.: 01014312584

email: marwasaber@aun.edu.eg marwasaber0607@gmail.com

\section{Introduction:}

NHL is the most prevalent hematologic malignancy worldwide. NHL ranks among the top ten most common malignancies accounting for $2.8 \%$ of all new cancer diagnoses globally [1]. In Egypt, NHL is the fifth most common cancer in both sexes, mostly with a late onset of the disease [2].
Notable group of patients with NHL may display a poor response to treatments with a true refractoriness or a transient response followed by early relapse. Rational of treatment depend on that most patients with relapsed or refractory NHL must receive second-line treatment sometimes followed by stem cell transplantation. Managing RIR NHL continues to be a challenge to the treating hemato-oncologist [3]. Anthracycline-based 
regimen in combination with rituximab, so called CHOP-R regimen (rituximab, cyclophosphamide, doxorubicin, vincristine and prednisone) has been the mainstay of treatment in NHL and mostly used as first line of treatment [4].

Multiple relapses are common for patients with NHL. Most relapses are detected within the first 2 years by the occurrence of clinical symptoms during the follow up and need second lines of treatment Relapsed cases can be clinically silent and is often diagnosed on routine follow-up. If clinical features and/or imaging findings suggest relapse, an excision biopsy should always be performed because R / R NHL mostly has poor prognosis [5].

Bendamustine is alkylating agent with multiple unique mechanisms of action and a lack of cross resistance with other alkylating agents, with multiple unique mechanisms of action. It is approved by the U.S. Food and Drug Administration (FDA) and the European Medicines Agency (EMA) for the treatment of patients with rituximab-refractory indolent B-cell NHL .In addition, it has recently been approved by Swissmedic for the first-line treatment of patients with FL in Switzerland. Approval by the FDA and EMA for firstline treatment of patients with FL and/or other indolent NHL. So bendamustine shown efficacy in refractory/relapsed setting suggesting the possible treatment option for patients failing conventional chemotherapy [6].

This work evaluated the efficacy and safety of using the Bendamustine as single agent in RIR NHL patients in an attempt to achieve better management of such condition.

\section{Patients and Methods:}

This study was carried out after being approved by the local ethics of South Egypt Cancer Institute, Assiut University, Egypt with informed consent was obtained from all patients.

This is a prospective, single arm, single center study carried out in Medical Oncology Department, South Egypt Cancer Institute, Assiut University in the period between January 2019 to December 2020 (two year) after fulfilling the coming inclusion and exclusion criteria and approval of the ethical committee.

Inclusion criteria included: age more than 18 years old, Eastern Cooperative Oncology Group (ECOG) performance status $\leq 2$, histological diagnosed as NHL according to WHO, patient documented to be RIR (patient who had appearance of new lesions after attainment of complete remission after 1year of treatment or after one or more line chemotherapy).

Exclusion criteria included: patient with age less than 18 year, patient with second primary malignancy, inadequate organ function that would compromise the safety of the patient or compromise the patient's ability to complete the study, patient with sever myelosuppression and pregnant or breast feeding women.

The patients were subjected to complete history taking include: personal history: as name, age, sex, residence, present history (as onset, course, duration type and stage of lymphoma), past history of any chronic or associated disorders, family history of lymphoma, or other general diseases, history of previous chemotherapy treatment and patient refused.

All patients were subjected to physical examination, routine laboratory tests included complete blood count (CBC), lactate dehadrogenase, liver function, bone marrow aspirate\& biopsy and virology and radiological examination before and after treatment (Computed tomography imaging of neck, chest and abdomen/Pelvis and PET with computed tomography).

As regard treatment protocol, every patient received bendamustine at dose of $120 \mathrm{mg} / \mathrm{m}^{2}$ (with $250 \mathrm{ml}$ saline over 60 minute in day 1 and 2 every 3 weeks for 3 cycle I. $V$ and reassess.

As regard patient assessment, tumor status was assessed clinically each cycle and every 3 cycles by CT imaging according to Response Evaluation Criteria in Solid Tumors (RECIST) [7]. Patients also assessed cycle by cycle for toxicity by clinical and basic laboratory investigations. Toxicity was reported according to the National Cancer Institute Common Terminology Criteria for Adverse Events (CTCAE) [8].

Follow up after complete response to treatment: history and physical examination and laboratory investigations every 4-6 month for 2 years, and radiological which included neck, chest, abdomen and pelvis CT scan with contrast every 4- 6 month for 2 years after completion of treatment.

\section{Statistical methods:}

All statistical calculations was done using SPSS (statistical package for the social science; SPSS Inc., Chicago, IL, USA) version 22. Data were statistically described in terms of mean \pm standard deviation $( \pm \mathrm{SD})$, or median and range when not normally distributed, frequencies (number of cases) and relative frequencies (percentages) when appropriate. OS and PFS were estimated using the Kaplan-Meier method.

\section{Results:}

Twenty five patient included in our study with patients characteristics are shown in Table 1 . In summary there were 14 males $(56 \%)$ and 11 females (44\%). the median age at diagnosis was 48 years. As regards diagnosis, there are 24 patient $(96 \%)$ patient had DLBCL (high grade), one (4\%) had follicular lymphoma (intermediate grade). According to stage, three patients (12\%) had stage $\amalg, 6$ patients $(24 \%)$ had stage $\amalg$ and 16 patients (64\%) had stage IV. Twelve patient $(48 \%)$ had B.M infiltration and $13(52 \%)$ had no infiltration. As regard to disease status, the study on eighteen $(72 \%)$ refractory patient, seven $(28 \%)$ relapsed patient with ten $(40 \%)$ of patient had low intermediate IPI, ten $(40 \%)$ of them had high-intermediate IPI and five $(20 \%)$ of them had high IPI.

Regard to previous lines; 25 (100\%) patients received CHOP, GDP used as second line in eleven $(44 \%)$ patients, ICE was used as second line in four 
(16\%) patients and as third line in six (24\%) patients, DHAP was used as second line in two (8\%) patients and as third line in three $(12 \%)$ patients and as fourth line in one $(4 \%)$ patients, Pegylated Liposomal Doxorubicin (calyx) -based use as third line in one (4\%) patient and fourth line in one (4\%) patient (figure1).

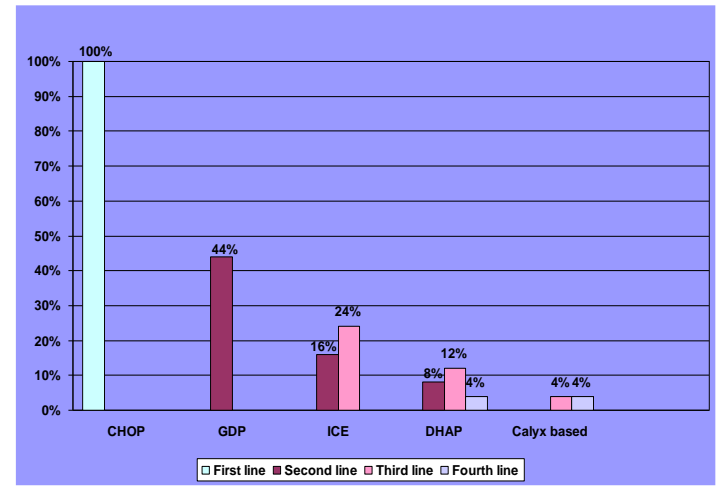

Figure (1) show previous lines of chemotherapy studied $25 \mathrm{R} \backslash \mathrm{R}$ NHL patients

Table 1: Patients characteristics of the studied 25 patients with R/R NHL

\begin{tabular}{|c|c|c|}
\hline Variable name & $\mathbf{N}$ & $(\%)$ \\
\hline $\begin{array}{l}\text { Follow up (months) } \\
\text { • Mean } \pm \text { SD } \\
\text { Age (vear) }\end{array}$ & \multicolumn{2}{|c|}{$10.24 \pm 6.12$} \\
\hline - Mean \pm SD & \multicolumn{2}{|c|}{$45.00 \pm 8.94$} \\
\hline $\begin{array}{ll}\text { Sex } & \\
\text { - Female } \\
\text { - Male }\end{array}$ & $\begin{array}{l}11 \\
14\end{array}$ & $\begin{array}{l}(44.0) \\
(56.0)\end{array}$ \\
\hline $\begin{array}{c}\text { Type of lymphoma } \\
\bullet \quad \text { DLBCL } \\
\bullet \quad \text { Follicular }\end{array}$ & $\begin{array}{c}24 \\
1\end{array}$ & $\begin{array}{r}(96.0) \\
(4.0)\end{array}$ \\
\hline $\begin{array}{cc}\text { Stage before treatment } \\
\text { - } & \text { Stage } 2 \\
\text { - } & \text { Stage } 3 \\
\text { - } & \text { Stage } 4\end{array}$ & $\begin{array}{c}3 \\
6 \\
16\end{array}$ & $\begin{array}{l}(12.0) \\
(24.0) \\
(64.0)\end{array}$ \\
\hline $\begin{array}{l}\text { Other comorbidity } \\
\text { - No } \\
\text { - Hypertension } \\
\text { - Diabetes } \\
\text { - Both } \\
\text { - Liver cirrhosis }\end{array}$ & $\begin{array}{l}19 \\
2 \\
2 \\
1 \\
1\end{array}$ & $\begin{array}{l}(76.0) \\
(8.0) \\
(8.0) \\
(4.0) \\
(4.0)\end{array}$ \\
\hline $\begin{array}{c}\text { BM infiltration } \\
\text { - Yes } \\
\text { - No }\end{array}$ & $\begin{array}{c}12 \\
13\end{array}$ & $\begin{array}{l}(48 \%) \\
(52 \%)\end{array}$ \\
\hline $\begin{array}{ll}\text { - } & \text { Low } \\
\text { - } & \text { Low-Intermediate } \\
\text { - } & \text { High-Intermediate } \\
\text { - } & \text { High }\end{array}$ & $\begin{array}{c}0 \\
10 \\
10 \\
5\end{array}$ & $\begin{array}{l}(0.0) \\
(40.0) \\
(40.0) \\
(20.0)\end{array}$ \\
\hline $\begin{array}{c}\text { Status of the patient at time } \\
\text { - Refractory } \\
\text { - Relapsed }\end{array}$ & $\begin{array}{c}\operatorname{g~Be} \\
18 \\
7\end{array}$ & $\begin{array}{l}\text { ustin } \\
(72.0) \\
(28.0)\end{array}$ \\
\hline
\end{tabular}

As regard treatment schedule and response rate there were 17 patients $(68 \%)$ received six cycle but 8 patient received only three cycle because of progression or death or referral to bone marrow transplantation (one case). with five patients (20\%) achieved CR, 10 patients $(40 \%)$ achieved PR, nine patients (36\%) had PD and one patient $(4 \%)$ had SD as shown in (Table 2). Overall survival was $72.0 \pm 9 \%$ at 6 months, $72.0 \pm 9 \%$ at 12 months, $66.0 \pm 10.0 \%$ at 18 months, and $66.0 \pm 10.0 \%$ at 24 month as shown in figure 2 and table 3 .

Table 2: Treatment schedule and patient's response studied patients

\begin{tabular}{|c|c|c|}
\hline Variable name & $\mathrm{N}$ & (\%) \\
\hline \multicolumn{3}{|c|}{ Number of cycle of Bendamustine } \\
\hline - Three cycles & 8 & (32.0) \\
\hline - Six cycles & 17 & $(68.0)$ \\
\hline \multicolumn{3}{|c|}{ Regularity of Bendamustine } \\
\hline - Regular & 24 & (96.0) \\
\hline - Irregular & 1 & $(4.0)$ \\
\hline \multicolumn{3}{|c|}{ Response after treatment by Bendamustine } \\
\hline - $\mathrm{CR}$ & 5 & (20) \\
\hline - $\quad \mathrm{PR}$ & 10 & (40) \\
\hline - $\mathrm{SD}$ & 1 & (4) \\
\hline - $\mathrm{PD}$ & 9 & (36) \\
\hline
\end{tabular}

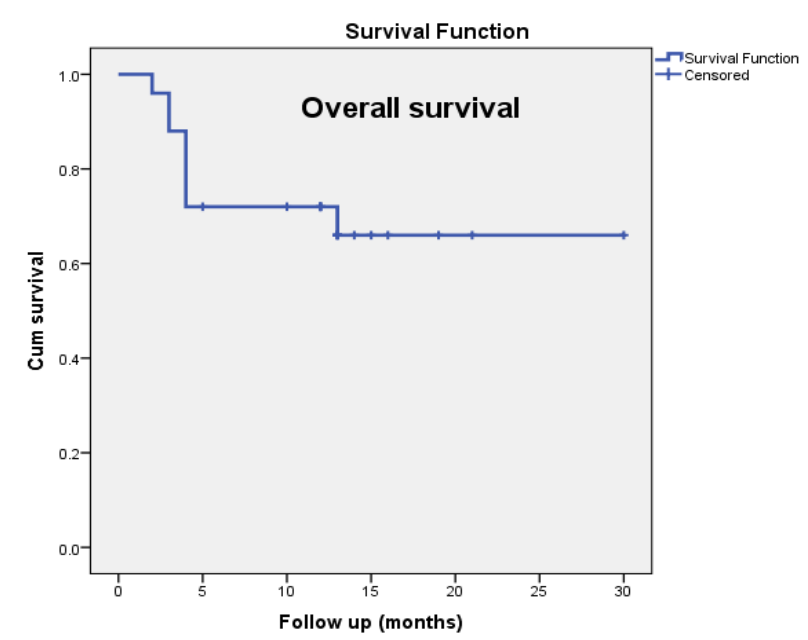

Figure (2) show Overall survival analyses (Kaplan -

Meier Estimation) was defined as the time from enrollment on the study to death from any cause or last follow up

Table 3: Clinical outcome, Overall survival analyses (Kaplan -Meier Estimation) of 25 patients with RIR NHL

\begin{tabular}{lc}
\hline OS & Estimate \pm SE \\
\hline At 6 months & $72.0 \pm 9.0 \%$ \\
At 12 months & $72.0 \pm 9.0 \%$ \\
At 18 months & $66.0 \pm 10.0 \%$ \\
At 24 months & $66.0 \pm 10.0 \%$ \\
\hline
\end{tabular}


PFS was $68 \pm 9.3 \%$ at 6 months, $63 \pm 9.7 \%$ at 12 months, $63 \pm 9.7$ at 18 months, and $63 \pm 9.7$ at 24 month as shown in Figure 3 and table 4.

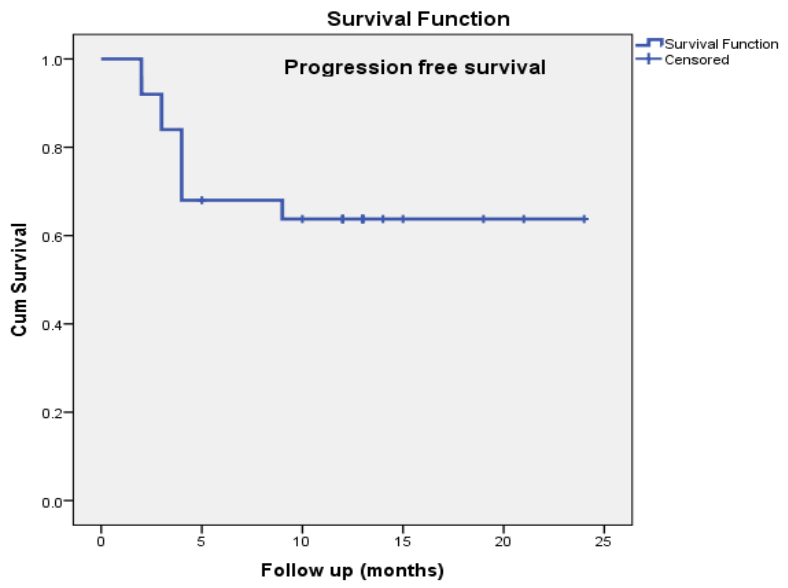

Figure (3) show Progression free survival analyses (Kaplan-Meier Estimation) that calculated from enrollment on the study to progression or death from NHL of 25 patients with RIR NHL

Table 4: Clinical outcome, Progression free survival (Kaplan-Meier Estimation) of 25 patients with RIR NHL

\begin{tabular}{lc}
\hline PFS & Estimate \pm SE \\
\hline At 6 months & $68.0 \pm 9.3 \%$ \\
At 12 months & $63.8 \pm 9.7 \%$ \\
At 18 months & $63.8 \pm 9.7 \%$ \\
At 24 months & $63.8 \pm 9.7 \%$ \\
\hline
\end{tabular}

As regard toxicity there were three $(12 \%)$ patients had no toxicity and $22(88 \%)$ patients developed toxicity. Concerning the toxicity, the main ones were haematological. Eighteen (72\%) patients developed haematological toxicity and four (16\%) developed nonhaematological toxicity. According to haematological toxicity, one patient (4\%) had thrombocytopenia G1-2. Four $(16 \%)$ patients had thrombocytopenia G3-4. Two (8\%) patients had neutropenia G1-2 (one case had febrile neutropenia). Seven (28\%) patients had neutropenia G3-4 (three cases had febrile neutropenia). Two $(8 \%)$ patients had anemia G1-2. Two (8\%) patients had anemia G3-4.

According to non-haematological toxicity. One (4\%) patient had fatigue grade $3-4$. One $(4 \%)$ patient had vomiting grade1-2. Two (8\%) patients had diarrhea grade 1-2 (Table 5).
Table 5: Toxicity to Bendamustine

\begin{tabular}{lcc}
\hline Variable name & $\mathrm{N}(25)$ & $\%$ \\
\hline Hematological Toxicity & & \\
• Thrombocytopenia G1-2 & 1 & $(4)$ \\
- Thrombocytopenia G3-4 & 4 & $(16)$ \\
- Neutropenia G1-2 & 2 & $(8)$ \\
- Neutropenia G3-4 & 7 & $(28)$ \\
- Anemia G1-2 & 2 & $(8)$ \\
- Anemia G3-4 & 2 & $(8)$ \\
Non-hematological Toxicity & & \\
- Fatigue G3-4 & 1 & $(4)$ \\
- Vomiting G1-2 & 1 & $(4)$ \\
- Diarrhea G1-2 & 2 & $(8)$ \\
No toxicity documented & $\mathbf{3}$ & $\mathbf{( 1 2 )}$ \\
\hline
\end{tabular}

\section{Discussion:}

Non-Hodgkin lymphoma is the most prevalent hematologic neoplasm. No standard treatment having been established for relapsed and refractory NHL [9]. Bendamustine is an alkylating novel agent with multiple unique mechanisms of action. It is approved by the U.S. Food and Drug Administration (FDA) and the European Medicines Agency (EMA) for the treatment of patients with rituximab-refractory indolent NHL [10].

In our study, (20\%) of patients had CR, (40\%) achieved PR so over all response rate(ORR) was $60 \%$ including $5 \mathrm{CR}, \mathrm{SD}$ in $(4 \%)$ and $(36 \%)$ achieved progression to treatment . this near result of Ohmachi et al [14] who made a multicenter, open-label, phase II study on 63 patients, the CR rate was $37.3 \%$, with $25.4 \%$ achieving PR so ORR $62.7 \%$ with $16.9 \%$ achieving stable disease, only $16.9 \%$ developing progressive disease, and $3.4 \%$ not evaluated that also detect good response with bendamustine. Also this near result of Heider et al [11] administered bendamustine at $120 \mathrm{mg} / \mathrm{m} 2$ on 2 successive days every 3 weeks to 52 patients with relapsed or refractory indolent lymphomas. Over all response rate response rate was $73 \%$, including 6 CRs $(11 \%)$. In contrast To Bremer et al [12] administered $60 \mathrm{mg} / \mathrm{m}^{2} / \mathrm{d}$ for 5 consecutive days to 102 patients with a variety of lymphoid malignancies, including 46 with lymphoplasmacytic lymphoma, and reported over all response rate of $82 \%$, with $15 \%$ CRs. These data were confirmed in the pivotal trial reported by Kahl et al [13] conducted on 100 patients with rituximab-refractory indolent NHL by using bendamustine at $120 \mathrm{mg} / \mathrm{m}^{2}$ on days 1 and 2 every 21 days for six cycles with patients received a median of three prior regimens including two prior rituximabcontaining regimens, $37 \%$ had received prior nucleoside analog therapy and $24 \%$ prior radioimmunotherapy

In our study, median for PFS was 12 months with range from (2-24) month and PFS after 12 months were $63.8 \%$ with median follow up 12 month. Several studies demonstrated almost nearer results as Kahl et al [13] who reported that the median DOR was 9.2 months and median PFS was 9.3 months. But Friedberg et al [15] study which reported that, median PFS was 8.3 months for the indolent histologies and 4.2 months for the 
transformed NHL. As well, Ohmachi et al [14] study which documented that at the median follow-up of 4.7 months (range, 0.5 to 19.2 months) with median PFS was 6.7 months. In our study median overall survival is 12months ranged (2-24) unlike Heider et al [11] who reported the median over-all survival time was 36 months (3-67) . Overall survival was $72.0 \%$ at 12 months and $66 \%$ at 24 month in our study.

In our study the main toxicity observed is hematological with one of them $(4 \%)$ had thrombocytopenia grade $1-2$, four $(16 \%)$ of them had thrombocytopenia grade $3-4$, two $(8 \%)$ patient had neutropenia grade 1-2 (one of them had febrile neutropenia), seven $(28 \%)$ patient had neutropenia grade 3-4 (three of them had febrile neutropenia), two $(8 \%)$ them had anemia grade $1-2$ and two $(8 \%)$ of them had anemia grade 3-4 Similar to our findings; Friedberg et al [15] who demonstrated that the main toxicity is haematological including neutropenia, anemia, and thrombocytopenia were primarily grade 1-2 in severity. Five episodes of febrile neutropenia were documented with $47 \%$ of patients experienced grade 3 to 4 neutropenia, but only $7 \%$ had febrile neutropenia. Grade 3 to 4 thrombocytopenia and grade 3 anemia were reported in $24 \%$ and $9 \%$ of patients, respectively. As well, Ohmachi et al [14] who demonstrated that the main toxicity observed with bendamustine treatment was reversible myelosuppression, including grade 3-4 neutropenia (72\%), leukopenia (65\%) and thrombocytopenia (16\%). Vacirca et al [16] study which reported that the most common hematologic adverse event was neutropenia, thrombocytopenia and anemia, each occurring in $44 \%$ of patients also. Grade 4 adverse events included febrile neutropenia in four patients $(7 \%)$, thrombocytopenia in three patients $(5 \%)$, and leukopenia in one patient (2\%). Grade 3 adverse events included neutropenia in 17 patients (29\%), thrombocytopenia in 10 patients $(17 \%)$, leukopenia in 13 patient $(22 \%)$ and anemia in 7 patients $(12 \%)$. Weidmann et al [17] who reported that hematological toxicity grade 3 and 4 was generally moderate $(8-13 \%$ of all treatment cycles) but in two patients, bendamustine had to be stopped due to prolonged grade 4 thrombo- and leukocytopenia.

In our study thrombocytopenia grade 1-2 were in $4 \%$, neutropenia grade $1-2$ were in $8 \%$, anemia grade 1 2 in $8 \%$ this in disagreement with Köppler et al [18] who documented that thrombocytopenia grade 1-2 were in $60 \%$, neutropenia grade $1-2$ were in $31 \%$ and anemia were in $82 \%$.

In our study three (12\%) patients had herpes infection who not take prophylaxis anti-viral. This similar to Friedberg et al [15] who demonstrated that three patients experienced documented grade 3 infections (herpes infection and pneumonia) with neutropenia.

In our study, non-hematological toxicities are low incidence with one (4\%) of them had fatigue grade 3-4, one $(4 \%)$ of them had vomiting grade1-2 and two (8\%) patient had diarrhea grade 1-2 this in agreement with Weidmann et al [17] who reported that subjective nonhematological toxicity was moderate to low with no grade 4 toxicity was observed with respect to nausea/emesis, fever, infections or diarrhea, and grade 3 toxicity for these side effects was low incidence $(<10 \%$ of all evaluated treatment cycles). In disagreement with Vacirca et al [16] who reported that non-hematological adverse events included anorexia, dehydration, fatigue, mucositis, shingles, and weight loss which not reported with our study.

Because bendamustine is an alkylating agent, secondary malignancies are a potential concern, however, no secondary malignancy reported in our study this in agreement with Weidmann et al [17] who reported that none of the patients had secondary malignancy. In disagreement with Friedberg et al [15] who demonstrated that secondary malignancies developed in three patients, all of whom eventually died.

In our study only three cases died because disease progression this similar to Ohmachi et al [14] who reported similar result with three deaths also occurred during the treatment period these were caused by disease progression, respiratory failure owing to tumor related airway compression, and hypotensive shock of unknown etiology but Vacirca et al [16] reported that four deaths occurred on during the study which included two patients who died from disease-related complications, one patient from disseminated herpes zoster, and one patient of unknown cause which was not matching with Weidmann et al study [17] which reported that none of the patients died.

\section{Conclusion:}

From our study we can conclude that Bendamustine is suggested to be an effective regimen in treatment of RIR NHL with high response rate and low toxicity profile.

\section{Limitation of our study}

Small sample size is obstacle in our study.

\section{Acknowledgements}

This paper is a part of master thesis. It was approved by ethical approval of institutional review board of South Egypt Cancer Institute, Assiut University.

Conflicts of interest: The authors declare no conflicts of interest regarding publishing this paper.

\section{References:}

1. Siegel RL, Miller KD, Jemal A, et al. Cancer statistics, 2020. CA Cancer J Clin. 2020 Jan;70(1):7-30.

2. Abdel-Fattah MM, Yassine OG. Non-Hodgkin's lymphomas in Alexandria, Egypt; incidence rates and trend study (1995-2004). Eur J Cancer Prev. 2007;16(5):479-85.

3. Al-Hamadani M, Habermann TM, Cerhan JR, et al. Non-Hodgkin lymphoma subtype distribution, geodemographic patterns, and survival in the US: A longitudinal analysis of the National cancer data Base from 1998 to 2011. Am J Hematol. 2015 
Sep;90(9):790-5.

4. Zhang Y, Dai Y, Zheng T, Ma S. Risk Factors of Non-Hodgkin Lymphoma. Expert Opin Med Diagn. 2011 Nov 1;5(6):539-550.

5. Glass B, Dohm AJ, Truemper LH, et al. Refractory or relapsed aggressive B-cell lymphoma failing (R)CHOP: An analysis of patients treated on the RICOVER-60 trial. Ann Oncol. 2017 Dec 1;28(12):3058-3064.

6. Cheson BD, Rummel MJ. Bendamustine: rebirth of an old drug. J Clin Oncol. 2009 Mar 20;27(9):1492-501.

7. Miyamoto K, Watanabe T, Kitahara H, et al. The Response Evaluation Criteria in Solid Tumors Can be Substituted for the International Workshop Criteria; Comparison Using Japan Clinical Oncology Group Study JCOG0203 for Untreated Indolent B-Cell Lymphoma. Blood 2016; 128(22): 4779.

8. Atkinson TM, Li Y, Coffey CW, et al. Reliability of adverse symptom event reporting by clinicians. Qual Life Res. 2012;21 (7):1159-1164.

9. Vacira JL, Tabbara IA, et al. Canadian Cancer Society's Advisory Committee on Cancer Statistics. Canadian Cancer Statistics 2015. Toronto, ON: Canadian Cancer Society; 2015.

10. Salles G, Duell J, González Barca E, et al. Tafasitamab plus lenalidomide in relapsed or refractory diffuse large B-cell lymphoma (LMIND): a multicentre, prospective, single-arm, phase 2 study. Lancet Oncol. 2020 Jul;21(7):978988.

11. Heider A, Niederle N. Efficacy and toxicity of bendamustine in patients with relapsed low-grade non-Hodgkin's lymphomas. Anticancer Drugs.
2001 Oct;12(9):725-9.

12. Bremer $\mathrm{K}$. High rates of long-lasting remissions after 5-day bendamustine chemotherapy cycles in pretreated low-grade non-Hodgkin's lymphomas. J Cancer Res Clin Oncol. 2002 Nov;128(11):603-9.

13. Kahl BS, Bartlett NL, Leonard JP, et al Bendamustine is effective therapy in patients with rituximab-refractory, indolent B-cell non-Hodgkin lymphoma: Results from a multicenter study. Cancer. 2010 Jan 1;116(1):106-14.

14. Ohmachi K, Niitsu N, Uchida T, et al. Multicenter Phase II Study of Bendamustine Plus Rituximab in Patients With Relapsed or Refractory Diffuse Large B-Cell Lymphoma. J Clin Oncol. 2013 Jun 10;31(17):2103-9.

15. Friedberg JW, Cohen $\mathrm{P}$, Chen $\mathrm{L}$, et al. Bendamustine in Patients with RituximabRefractory Indolent and Transformed NonHodgkin's Lymphoma: Results from a Phase II Multicenter, Single-Agent Study. J Clin Oncol. 2008 Jan 10;26(2):204-10.

16. Vacirca JL, Acs PI, Tabbara IA, et al. Bendamustine combined with rituximab for patients with relapsed or refractory diffuse large $B$ cell lymphoma. Ann Hematol. 2014 Mar;93(3):403-9.

17. Weidmann E, Kim SZ, Rost A, et al. Bendamustine is effective in relapsed or refractory aggressive non-Hodgkin's lymphoma. Ann Oncol. 2002 Aug;13(8):1285-9.

18. Köppler H, Heymanns J, Pandorf A, et al. Bendamustine plus mitoxantrone-A new effective treatment for advanced chronic lymphocytic leukaemia: Results of a phase I/II study. Leuk Lymphoma. 2004 May;45(5):911-3. 